\title{
Cells Attachment Property of PVA Hydrogel Nanofibers Incorporating Hyaluronic Acid for Tissue Engineering
}

\author{
Kyu-Oh Kim', Yaeko Akada', Wei Kai ${ }^{2}$, Byoung-Suhk Kim², Ick-Soo Kim² \\ ${ }^{1}$ Department of Bioscience and Textile Technology, Shinshu University, Nagano, Japan; ${ }^{2}$ Nano Fusion Technology Research Group, \\ Faculty of Textile Science and Technology, Shinshu University, Nagano, Japan. \\ Email:kbsuhk@yahoo.com, kim@shinshu-u.ac.jp
}

Received May $20^{\text {th }}, 2011$; revised June $20^{\text {th }}, 2011$; accepted July $20^{\text {th }}, 2011$.

\begin{abstract}
In this work, we report the fabrication and cell affinity studies of the poly (vinyl alcohol) (PVA)/hyaluronic acid (HA) cross-linked nanofibers via electrospinning and post cross-linking. FT-IR and TGA analysis demonstrate that HA is not influenced by acid environment such as HCl vapor during cross-linking, and well incorporated into PVA nanofibers. Swelling behavior and cell adhesion of the PVA/HA hydrogel nanofibers are investigated and compared with pure PVA hydrogel nanofibers. It is expected that the nanofibrous PVA/HA hydrogel fibers could be a promising scaffold for cell culture and tissue engineering applications.
\end{abstract}

Keywords: Nanofiber, Electrospinning, Hydrogel, Poly(Vinyl Alcohol), Hyaluronic Acid, Cell Affinity

\section{Introduction}

Tissue-engineered skin has enormous potential that is only just beginning to be realized and has delivered considerable benefits to patients with chronic wounds. A number of people need skin grafts due to dermal wounds caused by fire, heat, electricity, chemicals, ultraviolet, nuclear energy, or diseases. In the case of wounds that extend entirely through the dermis, such as full-thickness burns or deep ulcers, many skin substitutes such as xenograft, allograft, isograft and autograft have been employed for wound healing. However, these approaches have disadvantages, which are high cost, the limited availability of skin grafts in severely burned patients, and problems of disease transmission and immune response [1-3]. Currently, various carrier dressing of skin repair product have being explored to improve factors such as ease of use, cost, transportation and variables for both the clinician and the patients for achieving skin repair made into bovine collagen [4], thin polymer containing acid functional groups using the plasma polymerization [5]. As the other remedy, the nanofiber technology have great potential of this skin dressing by advantages of the nanofiber such as similar morphology to a natural extracellular matrix (ECM), high surface-area, excellent air-perme- ability and low cost. To make the electrospun hydrogel nanofibers as a wound dressing, we used two polymers, hyaluronic acid (HA) and poly (vinyl alcohol) (PVA). First, HA having the unique hygroscopy could play a bioactive role in wound healing by creating a swollen macro- and micro-environment [6,7]. PVA was known to get excellent biological properties, good physical property as well as to form the hydrogel by chemical gelation via $\gamma$-ray or electron-beam irradiation $[8,9]$ using glutaraldehyde (GA) as a cross-linker and by physical gelation via freezing-thawing cycles [10]. However, the fabrication of PVA cross-linked nanofibers via electrospinning process has a difficulty due to rapidly increase in solution viscosity, if GA agent is used [11]. This disadvantage caused to be poor electrospinnability and was also hard to control. To overcome these problems, in this study, we use post cross-linking method of the electrospun PVA/HA nanofibers using $\mathrm{GA}$ and $\mathrm{HCl}$ as the cross-linker and catalyst, respectively, and report enhanced bioactivity of the resultant PVA/HA hydrogel nanofibers, compared to pure PVA hydrogel nanofibers. The motivation of this study is to develop and optimize the novel PVA/HA hydrogel nanofibers. The cell culture using MC3T3-E1 is also conducted to assess the viability and potential application of this material as a scaffold. 


\section{Experimental Section}

\subsection{Materials}

Poly (vinyl alcohol) $(\mathrm{PVA})$ (degree of hydrolysis $=88 \%$, degree of polymerization (DP) $\approx 1700$ ) was obtained from Kuraray Co. Ltd., Japan. Glutaraldehyde (GA, 50\% in water) was purchased from Sigma-Aldrich, USA. Hyaluronic acid (HA, SHANDONG FREDA BIOPHARM Co., China) was used as received. Hydrochloric acid (purity, 37\%) and ethanol (purity, 99.5\%) were purchased from Wako Chem. Co., Japan. All materials were used as-received without further purification.

\subsection{Electrospinning}

A high-voltage power supply (Har-100*12, Matsusada Co., Japan), capable of generating voltages up to $100 \mathrm{kV}$, was used as a source of electric filed. The pure PVA and PVA/HA solutions were supplied through a $5 \mathrm{ml}$ plastic syringe attached to a capillary tip with an inner diameter of $0.6 \mathrm{~mm}$. The copper wire connected to a positive electrode (anode) was inserted into each polymer solution, and a negative electrode (cathode) was attached to a metallic collector. The applied voltage was fixed at $14 \mathrm{kV}$. The distance between the capillary tip and the collector was fixed to be $15 \mathrm{~cm}$. All solutions were electrospun onto a rotating metallic collector at room temperature under identical conditions.

\subsection{Cross-Linking of Electrospun PVA and PVA/HA Nanofibers}

In order to achieve the cross-linking of PVA nanofibers, GA as a cross-linker was added to both pure PVA and PVA/HA solutions, respectively. The PVA/GA solutions (molar ratios of GA and PVA were 3.4/1.0, 6.9/1.0 and 10.3/1.0) were dissolved in distilled water at $50^{\circ} \mathrm{C}$ overnight. On the other hand, the PVA/HA/GA solutions (molar ratios of GA and PVA were 8.2/1.0, 16.5/1.0 and 24.8/1.0) in the presence of HA were dissolved in the mixed water/ethanol $(9 / 1, \mathrm{v} / \mathrm{v})$ solvent at $50^{\circ} \mathrm{C}$ overnight to improve the solubility HA in PVA/GA solution. Here, the concentration of PVA solution was fixed at $12 \mathrm{wt} \%$. The weight ratio of PVA and HA was 5/1. Afterwards, the obtained electrospun nanofibers were exposed to $\mathrm{HCl}$ vapor, which was produced in the desiccators at $30^{\circ} \mathrm{C}$. The exposure time (10 s, $30 \mathrm{~s}$ and $60 \mathrm{~s}$ ) of acid treatment and molar ratio of GA and PVA were varied. After cross-linking, the samples were found to shrink slightly during the treatment of $\mathrm{HCl}$ vapor, and used carefully after vacuum-drying at room temperature.

\subsection{Characterization}

To identify the cross-linking of PVA/GA and the incorporation of HA, FT-IR analysis was carried out using an
IRPrestige-21 (Shimadzu Co., Japan). In order to determine the amount of HA presenting in the cross-linked PVA/HA nanofibers, thermogravimetric analysis (TGA) (TG/DTA6200, Seiko Instruments Inc. Japan) was carried out by heating from room temperature to $600^{\circ} \mathrm{C}$ under a continuous nitrogen purge of $20 \mathrm{~mL} / \mathrm{min}$. The morphologies of electrospun PVA/HA hydrogel nanofibers were characterized using scanning electron microscopy (SEM, S-3000N HITACHI, Japan) on samples sputtered with Pd-Pt. The fiber diameter and its distribution were determined by using image J (ImageJ v1.41, Wayne Rasband National institutes of Health, USA) software.

\subsection{Swelling Behavior}

After measuring the initial size and weight, dried electrospun fibrous membranes were immersed into distilled water for 5 days at room temperature to attain an equilibrium swelling state. Before measuring the size and weight of the swollen samples, the excess amounts of waters onto the surface were carefully removed by blotting with filter paper. The swelling ratio was calculated as follows [12]:

$$
\text { Swelling ratio }(g / g)=\frac{W_{s}-W_{d}}{W_{d}}
$$

where $W_{s}$ and $W_{d}$ are the weights of swollen and dried samples, respectively.

\subsection{Cell Culture}

The pure PVA and PVA/HA hydrogel nanofibers (collected on round cover glass slips of $15 \mathrm{~mm}$ in diameter) were immersed in $80 \%$ ethanol for 2 hrs for sterilization purposes. The hydrogel nanofibers were washed with phosphate buffered saline (PBS) thrice followed by culture medium thrice to eliminate any residual ethanol. MC3T3-E1 osteoblast-like cells, which were obtained from the RIKEN Cell Bank (Tsukuba, Japan), were cultured until passage 7 and seeded on pure PVA and PVA/ HA hydrogel nanofibers at a cell concentration of $3 \times 10^{4}$ cells/well. Cells were incubated at $37^{\circ} \mathrm{C}$, in a $5 \% \mathrm{CO}_{2}$ atmosphere incubator, using $\alpha$-modified minimal essential medium ( $\alpha$-MEM; GIBCO). The medium comprised of $10 \%$ heat-inactivated fetal bovine serum (FBS), 100 $\mathrm{U} / \mathrm{ml}$ penicillin, $100 \mathrm{U} / \mathrm{ml}$ streptomycin and $0.1 \% \beta$-glycerophosphate was used to induce osteoblastic differentiation. For all cell investigations, cells cultured on TCDs (tissue culture dishes, high-grade polystyrene $\mathrm{Nunc}^{\mathrm{TM}}$ Dishes, Thermo Fisher Scientific, Denmark) were evaluated as controls. The medium was changed every two days to ensure that there was an adequate supply of nutrients present in the culture plate. The hydrogel nanofibrous scaffolds, which were inoculated with MC3T3-E1 (cell culture for 1, 6 and $24 \mathrm{hrs),} \mathrm{were} \mathrm{fixed} \mathrm{in} \mathrm{10 \%} \mathrm{for-}$ 
maldehyde solution overnight at $4^{\circ} \mathrm{C}$. Next, the scaffolds were dehydrated in a series of 5 cycles ( 1 cycle: $5 \mathrm{~min}$ ) with increasing concentrations of ethanol: $50 \%, 60 \%$, $70 \%, 80 \%, 90 \%$ and $100 \%$ (twice) respectively. Finally, the samples were freeze-dried overnight and scanning electron microscopy (SEM, S-3000N HITACHI, Japan) analysis was done to view the morphology of the attached cells on the nanofiber scaffolds.

\subsection{Cell Adhesive}

Cells were seeded and cultured as under the same conditions in "Cytotoxicity assay and live/dead cell staining" section. Cells in culture medium were counted $(\mathrm{Nm})$ after 1, 3, 6, and $24 \mathrm{hrs}$ of incubation. The cell adhesion ratio for each condition was calculated using the following equation:

$$
\text { Adhesion ratio }(\%)=\left(1-\mathrm{Nm} / 3.0 \times 10^{4}\right) \times 100
$$

All data reported were the mean of three examinations.

\section{Results and Discussion}

\subsection{FT-IR Study}

Figure 1 shows FT-IR spectra of pure PVA nanofibers, pure PVA cross-linked nanofibers (molar ratio of GA and PVA 10.3:1.0), and PVA/HA cross-linked nanofibers (molar ratio of GA and PVA 24.8:1.0). All spectra show characteristic PVA bands at hydroxyl group (3300 $\mathrm{cm}^{-1}$ ), alkyl group (2906 $\left.-2908 \mathrm{~cm}^{-1}\right)$ and acetyl group $\left(1730 \mathrm{~cm}^{-1}\right)$, respectively. After cross-linking, the intensity of hydroxyl group at $3685-3000 \mathrm{~cm}^{-1}$ was relatively reduced, compared with pure PVA nanofibers, indicating the successful cross-linking reaction between - $\mathrm{OH}$ group in PVA and -CHO in GA. Furthermore, assuming that the $\mathrm{C}=\mathrm{O}$ contents, whose intensity do not change from the region between $1788-1639 \mathrm{~cm}^{-1}$ during cross-linking reaction, are set to constant intensity [13], it was estimated that loss contents of hydroxyl group in the PVA cross-linked nanofibers (molar ratio of GA and PVA 10.3:1.0) was about 5.84\%, which was ascribed to a possible formation of acetal bridges from $\mathrm{C}-\mathrm{H}$ stretching related to aldehyde group [14]. In addition, in case of the PVA/HA cross-linked nanofibers, N-H bending was clearly appeared at near $1635 \mathrm{~cm}^{-1}$, suggesting that HA was not influenced by acid environment such as $\mathrm{HCl}$ vapor during cross-linking.

\subsection{TGA Study}

Figure 2 shows TGA curves of pure PVA nanofibers, pure PVA cross-linked nanofibers (molar ratio of GA and PVA 10.3:1.0), and PVA/HA cross-linked nanofibers (molar ratio of GA and PVA 24.8:1.0). Firstly, pure PVA showed three-step degradation behaviors. That is, the first degradation step below $150^{\circ} \mathrm{C}$ was aware of the removal of moisture, physisorbed, and chemisorbed water molecules. The second degradation step was associated with the degradation of PVA backbond. The third degradation step around $600^{\circ} \mathrm{C}$ was depicted to the degradation of vinyl acetate group of PVA. Majority weight loss of pure PVA nanofibers was occurred around $300^{\circ} \mathrm{C}$, while pure PVA cross-linked nanofibers showed higher

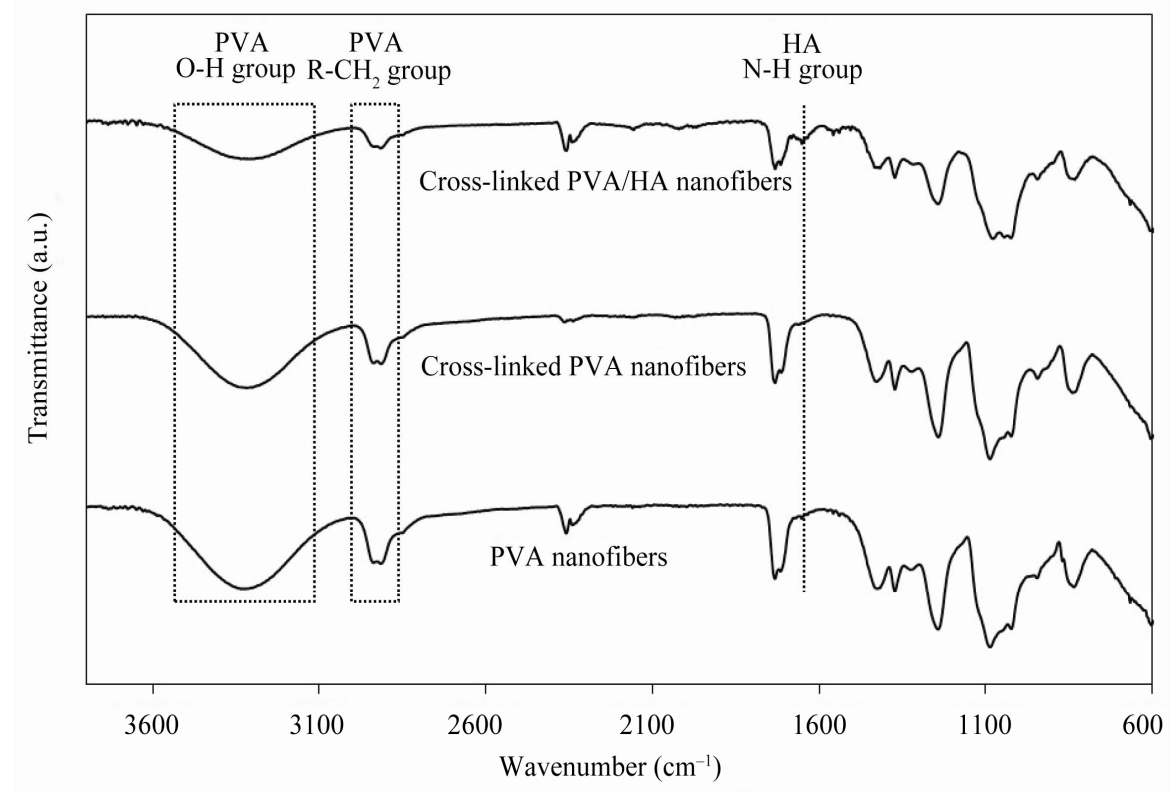

Figure 1. FT-IR spectra of pure PVA nanofibers, pure PVA cross-linked nanofibers (molar ratio of GA and PVA 10.3:1.0), and PVA/HA cross-linked nanofibers (molar ratio of GA and PVA 24.8:1.0). The concentration of PVA solution is 12 wt\%. 


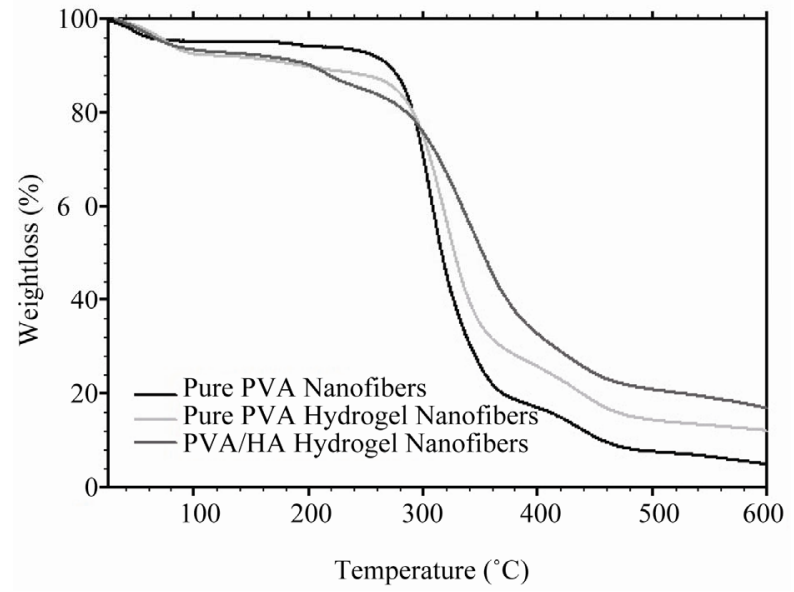

Figure 2. TGA curves of pure PVA nanofibers, pure PVA cross-linked nanofibers (molar ratio of GA and PVA 10.3:1.0), and PVA/HA cross-linked nanofibers (molar ratio of GA and PVA 24.8:1.0). The concentration of PVA solution is $12 \mathrm{wt} \%$.

thermal stability than pure PVA due to cross-linking [15]. Moreover, the PVA/HA cross-linked nanofibers exhibited weight loss of $4 \%$ at about $215^{\circ} \mathrm{C}$ by the decomposition of hyaluronic acid, suggesting that HA was well incorporated into the PVA cross-linked nanofibers. The weight residue of pure PVA nanofibers around $600^{\circ} \mathrm{C}$ was ca. $5.1 \%$ due to the various carbonaceous matters, whereas both pure PVA and PVA/HA cross-linked nanofibers showed much higher char values of $12.2 \%$ and $17.0 \%$ than that of pure PVA, respectively, due to the alkyl group of GA.

\subsection{Morphologies}

Electrospinnability was strongly influenced by solvents and solution viscosity during electrospinning process [16-21]. Here, mixed solvent (water/ethanol $=9 / 1, \mathrm{v} / \mathrm{v})$ was used in order to improve the solubility and the corresponding electrospinning of the PVA/HA solution. Figure 3 shows SEM images of pure PVA (a), PVA/GA (b), PVA/HA (c), and PVA/HA/GA (d) electrospun nanofibers before cross-linking. As evidenced by SEM analysis, the fiber diameters were regular with an average diameter of approximately $430 \pm 50 \mathrm{~nm}, 400 \pm 60 \mathrm{~nm}$, $200 \pm 50 \mathrm{~nm}, 180 \pm 50 \mathrm{~nm}$, and its distribution was narrower. It can be seen that GA did not influence the diameters of the resulting nanofibers, whereas the incorporation of $\mathrm{HA}(\mathrm{PVA} / \mathrm{HA}$ and $\mathrm{PVA} / \mathrm{HA} / \mathrm{GA}$ ) resulted in smaller diameters than that of pure PVA nanofiber due to an enhanced conductivity by hyaluronic acid. Furthermore, the investigation of the fiber morphologies and the pores in aqueous media are important for applications as wound dressings.

After cross-linking, we have also studied the changes in the morphologies and swelling behavior of both pure PVA and PVA/HA cross-linked nanofibers after immersing in distilled water for 72 hrs. As seen in Figures 4 and 5 , it was observed that the fiber morphologies of
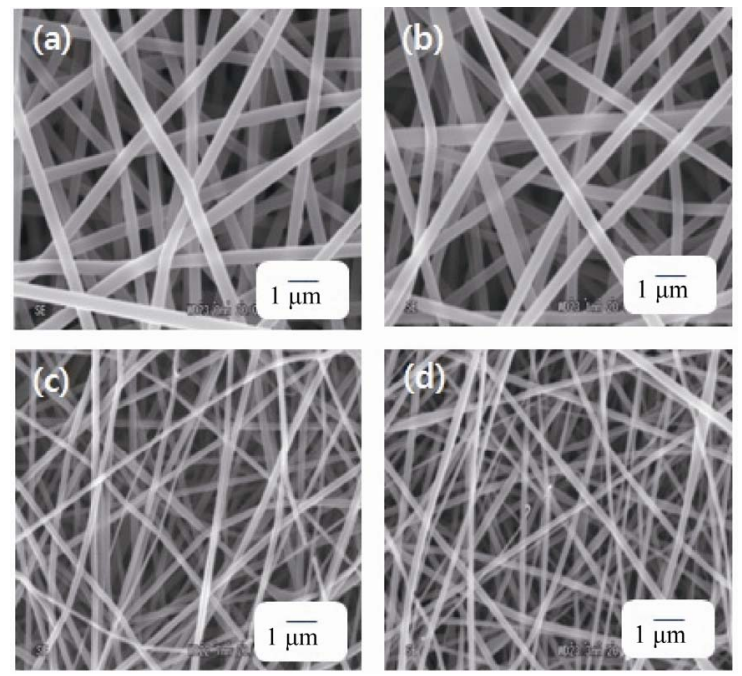

Figure 3. SEM images of pure PVA (a), PVA/GA (b), molar ratio of GA and PVA 10.3:1.0), PVA/HA (c), and PVA/ HA/GA (d, molar ratio of GA and PVA 24.8:1.0) nanofibers before cross-linking. The concentration of PVA solution is $12 \mathrm{wt} \%$.

(a)

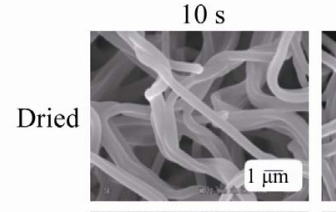

Cross-linking time
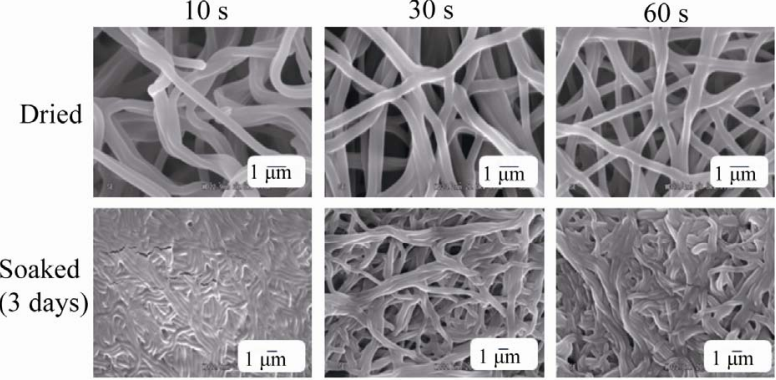

(b)

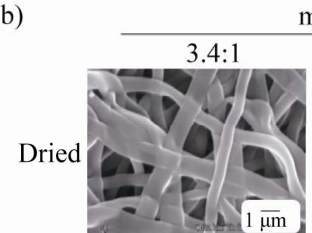

molar ratio of GA:PVA
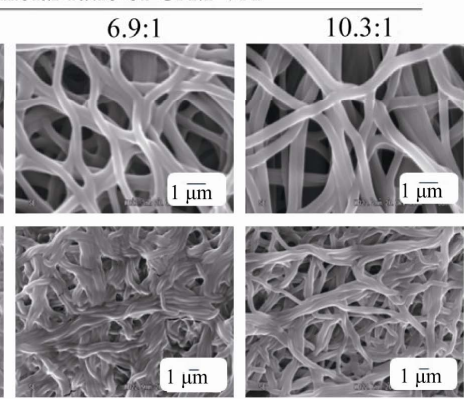

Figure 4. SEM images of PVA hydrogel nanofibers with different cross-linking time (a, at constant molar ratios of GA and PVA of 10.3:1.0) and various molar ratios of GA and PVA (b, at constant soaking time of $30 \mathrm{~s}$ ) before and after soaking in distilled water for 3 days. 


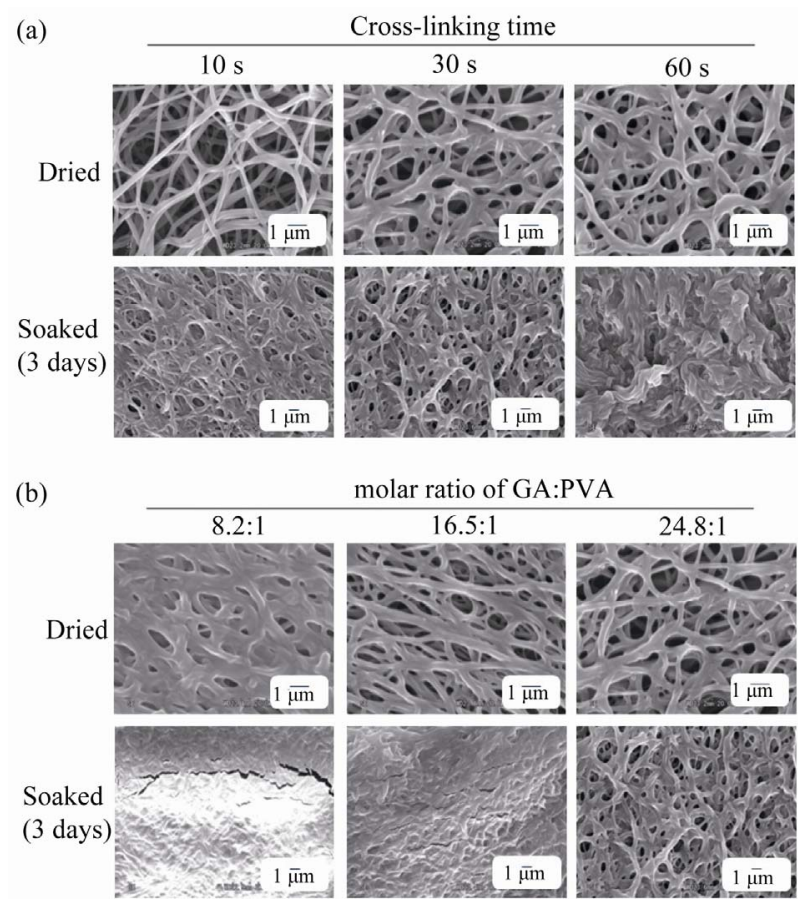

Figure 5. SEM images of PVA/HA hydrogel nanofibers with different cross-linking time (a, at constant molar ratios of GA and PVA of 24.8:1.0) and various molar ratios of GA and PVA (b, at constant soaking time of $30 \mathrm{~s}$ ) before and after soaking in distilled water for 3 days.

pure PVA and PVA/HA hydrogel nanofibers soaked in distilled water was strikingly different depending on the cross-linking time and the GA concentration (that is, the cross-linking density). The optimum cross-linking time and GA concentration on both pure PVA and PVA/HA hydrogel nanofibers were $30 \mathrm{sec}$ and $30 \mu \mathrm{m}$ (corresponding to molar ratio of GA and PVA 16.5:1.0), respectively, showing clear fibrous morphologies. Obviously, shorter cross-linking time and lesser GA concentration did not maintain fiber morphologies after soaking in distilled water and then resulted in fused film-like morphologies, due to an incomplete cross-linking.

\subsection{Swelling Behavior}

Hydrogels are soft and wet and look like a solid material, but are capable of undergoing large deformation [22,23], and thus are often used as swelling controlled-release devices, for which absorption of water leads to polymer expansion, which directly affects the diffusivity of drugs. The degree of swelling is related to the ability of solute molecules to move through the gels networks and the diffusion of fluid in the polymer as well as its macromolecular relaxation [24]. Figure 6 shows swelling ratio of pure PVA and PVA/HA hydrogel nanofibers as a function of soaking time at room temperature. In principle, the swelling extent of hydrogels is determined by a ba-

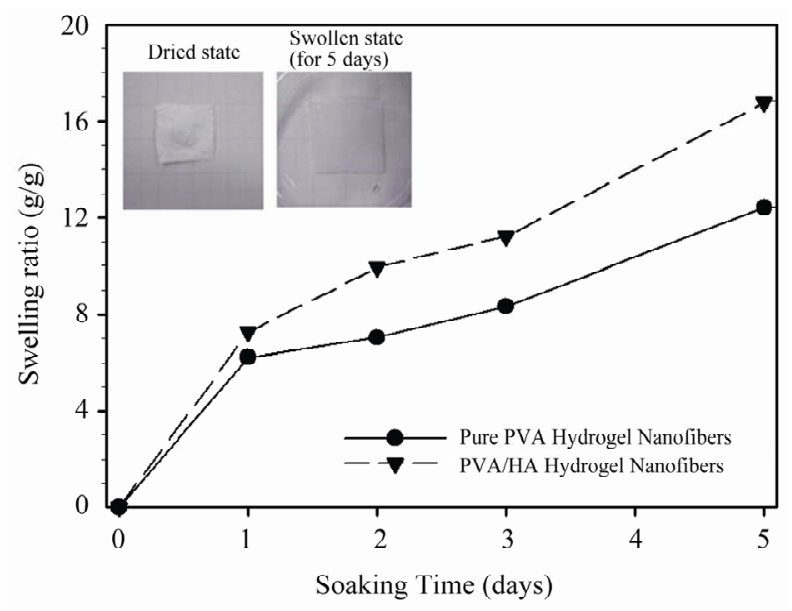

Figure 6. Swelling ratio of pure PVA hydrogel (molar ratio of GA and PVA 10.3:1.0) and PVA/HA hydrogel (molar ratio of GA and PVA 24.8:1.0) nanofibers as a function of soaking time at room temperature. The concentration of PVA solution is $12 \mathrm{wt} \%$.

lance between the driving force of an exothermic mixing enthalpy $\left(\Delta \mathrm{H}_{\text {swell }}<0\right)$, resulting from favorable interact tions between hydrophilic groups and water molecules, and a mediating loss of polymer chain conformation entropy $\left(\Delta \mathrm{S}_{\text {swell }}<0\right)$ during the same swelling. This behavior is well described by Flory-Rehner theory [25]. As seen in Figure 6, the pure PVA and the PVA/HA hydrogel nanofibers reached swelling ratios of ca. 6.2 and ca. 7.2 at the soaking time of $1 \mathrm{hr}$, indicating a fast swelling via a capillary force due to the nanoporous structures, and then gradually increased as increasing soaking time. Moreover, the swelling ratio of the PVA/ HA hydrogel nanofibers showed always higher values than those of pure PVA hydrogel nanofibers because of a strong hydrophilic HA. We could also see that the hydrogel nanofibers became transparent after swelling (see inset in Figure 6), indicating the water molecules diffusing into the sample to hydrate the polar/hydrophilic groups, leading to volumetric expansion that comes to an equilibrium swelling ratio. Evidently, the size of swollen sample also became larger, compared to the dried sample.

\subsection{Cell Adhesion and Its Morphology}

HA can directly communicate with proteins and cells present in tissues if HA is contained onto the surface of matrix well [26-29]. The adhesion ratio of MC3T3-E1 cells [30] on the pure PVA and PVA/HA hydrogel nanofibers was shown in Figure 7. Comparison of initial adhesion ratio (at $0 \mathrm{hr}$ ) for the PVA hydrogel nanofibers in the presence and absence of HA has probed that HA containing PVA hydrogel nanofibers showed relatively higher adhesion ratio, indicating that the PVA/HA hy- 
drogel nanofibers could acquire excellent interfacial biocompatibility. Moreover, they quickly reached as high as about $100 \%$ after 6 hrs' cultivation without difference. For cell spreading study, Figure 8 shows SEM images of MC3T3-E1 grown onto TCD, pure PVA and PVA/HA hydrogel nanofibers with various cell-culture times. It clearly appeared that MC3T3-E1 cells were well adhered to the PVA/HA hydrogel nanofibers unlike pure PVA hydrogel nanofibers, which is also well coincided with the results of adhesion ratio, probably due to the remarkable lower toxicity of HA. Also, it could be obviously seen that vigorous cell migration occurred on PVA/HA hydrogel nanofibers and a number of microvilli on the surfaces of nanofiber webs can be observed due to probably specific interactions between HA and cells. After

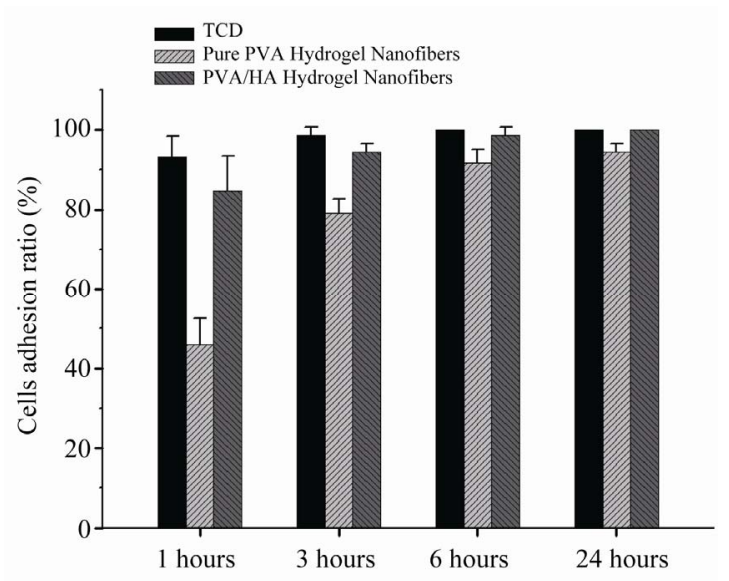

Figure 7. Adhesion ratio of MC3T3-E1 cells cultured on TCD, pure PVA hydrogel (molar ratio of GA and PVA 10.3:1.0), and PVA/HA hydrogel (molar ratio of GA and PVA 24.8:1.0) nanofibers. The concentration of PVA solution is $12 \mathrm{wt} \%$.

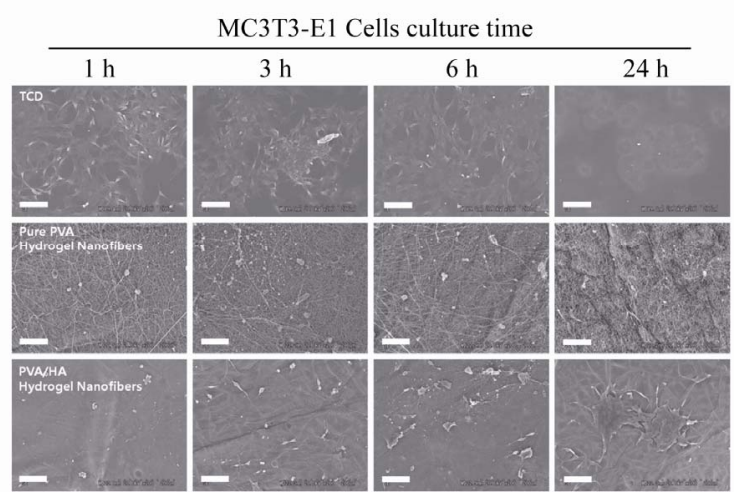

Figure 8. SEM images of MC3T3-E1 cells cultured on TCD, pure PVA hydrogel (molar ratio of GA and PVA 10.3:1.0), and PVA/HA hydrogel (molar ratio of GA and PVA 24.8:1.0) nanofibers as a function of cell-culture time. The concentration of PVA solution is $12 \mathrm{wt} \%$. The scale bar indicates $100 \mathrm{~nm}$. the cell culture for $24 \mathrm{hr}$, in particular, MC3T3-E1 cells grown onto PVA/HA hydrogel nanofibers became huge cells via cell-cell interactions, suggesting an enhanced cell migration for cell organization.

\section{Conclusions}

We have successfully prepared the pure PVA and PVA/ HA cross-linked nanofibers via electrospinning and post cross-linking. FT-IR and TGA analysis demonstrated that HA was not influenced by acid environment such as $\mathrm{HCl}$ vapor during cross-linking, and also well incorporated into PVA nanofibers. As evidenced by SEM analysis, the fiber diameters of the pure PVA, PVA/GA, PVA/ $\mathrm{HA}, \mathrm{PVA} / \mathrm{HA} / \mathrm{HA}$ electrospun nanofibers before crosslinking were regular with an average diameter of approximately $430 \pm 50 \mathrm{~nm}, 400 \pm 60 \mathrm{~nm}, 200 \pm 50 \mathrm{~nm}, 180 \pm$ $50 \mathrm{~nm}$, respectively. It was found that the fiber morphologies of pure PVA and PVA/HA hydrogel nanofibers soaked in distilled water was strikingly different depending on the cross-linking time and the GA concentration (that is, the cross-linking density). The pure PVA and the PVA/HA hydrogel nanofibers reached swelling ratios of ca. 6.2 and ca. 7.2 at the soaking time of $1 \mathrm{hr}$, indicating a fast swelling via a capillary force. The swelling ratio of the PVA/HA hydrogel nanofibers showed always higher values than those of pure PVA hydrogel nanofibers because of a strong hydrophilic HA. Comparison of initial adhesion ratio (at $0 \mathrm{hr}$ ) for the PVA hydrogel nanofibers in the presence and absence of HA has probed that HA containing PVA hydrogel nanofibers showed relatively higher adhesion ratio, indicating that the PVA/HA hydrogel nanofibers have an excellent interfacial biocompatibility.

\section{Acknowledgements}

The authors acknowledge the support of Shinshu University Global COE Program "International Center of Excellence on Fiber Engineering".

\section{REFERENCES}

[1] M. Sheila, "Progress and Opportunities in Tissue Engineering of Skin', Nature Insights," Nature, Vol. 445, No. 7130, 2007, pp. 874-880.

[2] B. L. Seal, T. C. Otero and A. Panitch, "Polymeric Biomaterials for Tissue and Organ Regeneration," Materials Science and Engineering, Vol. 34, No. 4-5, 2001, pp. 147-230. doi:10.1016/S0927-796X(01)00035-3

[3] J. J. Marler, J. Upton, R. Langer and J. P. Vacanti, "Transplantation of Cells in Matrices for Tissue Regeneration," Advanced Drug Delivery Reviews, Vol. 33, No. $1-2,1998$, pp. 165-182. doi:10.1016/S0169-409X(98)00025-8

[4] R. E. Horch, M. Debus, G. Wagner and G. B. Stark, "Cultured Human Keratinocytes on Type I Collagen 
Membranes to Reconstitute the Epidermis," Tissue Engineering, Vol. 6, No. 1, 2000, pp. 53-67. doi:10.1089/107632700320892

[5] R. M. France, R. D. Short, R. A. Dawson and S. Macneil, "Attachment of Human Keratinocytes to Plasma CoPolymers of Acrylic Acid/Octa-1,7-Diene and Allyl Amine/Octa-1,7-Diene," Journal of Materials Chemistry, Vol. 8, 1998, pp. 37-42. doi:10.1039/a705098d

[6] J. F. Kennedy, G. O. Phillips, P. A. Williams and V. Hascall, In: Hyaluronan Ed., "Functions of Hyaluronan in Wound Repair," Woodhead Publishing Ltd., Cambridge, , 2002, pp. 147-156.

[7] W. Y. J. Chen and G. Abatangelo, "Function of Hyaluronan in Wound Repair," Wound Repair and Regeneration, Vol. 7, No. 2, 1999, pp. 79-89. doi:10.1046/j.1524-475X.1999.00079.x

[8] T. Hatakeyama, A. Yamauchi and H. Hatakeyama, "Studies Bound Water in Poly(Vinyl Alcohol)," European Polymer Journal, Vol. 20, No. 1, 1984, pp. 61-64. doi:10.1016/0014-3057(84)90223-4

[9] N. A. Peppas and E. W. Merrill, "Poly (Vinyl Alcohol) Hydrogels: Reinforcement of Radiation-Crosslinked Networks by Crystallization," Journal of Polymer Science, Polymer Chemistry Edition, Vol. 14, No. 2, 1976, pp. 441-457. doi:10.1002/pol.1976.170140215

[10] M. Liu, R. Cheng and R. Qian, "Investigation of Swelling Property of Poly (Vinyl Alcohol) Hydrogel," Acta Polymerica Sinica, Vol. 1, No. 2, 1996, pp. 234-239.

[11] C. Tang, C. D. Saquing, J. R. Harding and S. A. Khan, "In situ Cross-Linking of Electrospun Poly(vinyl alcohol) Nanofibers," Macromolecules, Vol. 43, No. 2, 2010, pp. 630-637. doi:10.1021/ma902269p

[12] J. H. Pang, H. B. Zhang, X. F. Li and Z. H. Jiang, "Novel Wholly Aromatic Sulfonated Poly (Arylene Ether) CoPolymers Containing Sulfonic Acid Groups on the Pendants for Proton Exchange Membrane Materials," Macromolecules, Vol. 40, No. 26, 2007, pp. 9435-9442. doi:10.1021/ma070080s

[13] P. Alexy, D. Kachova, M. Krsiak, D. Bakos and B. Simkova, "Poly (Vinyl Alcohol) Stabilisation in ThermoPlastic Processing," Polymer Degradation and Stability, Vol. 78, 2002, pp. 21-413. doi:10.1016/S0141-3910(02)00177-5

[14] H. S. Mansur, C. M Sadahira, A. N. Souza and A. A. P. Mansur, "FTIR Spectroscopy Characterization of Poly (vinyl alcohol) Hydrogel with Different Hydrolysis Degree and Chemically Crosslinked with Glutaraldehyde," Materials Science and Engineering: C, Vol. 28, No. 4, 2008, pp. 539-548. doi:10.1016/j.msec.2007.10.088

[15] C. H. Chena, F. Y. Wang, C. F. Mao, W. T. Liao and C. D. Hsieh, "Studies of Chitosan: II. Preparation and Characterization of Chitosan/Poly(Vinyl Alcohol)/Gelatin Ternary Blend Films," International Journal of Biological Macromolecules, Vol. 43, No. 1, 2008, pp. 37-42. doi:10.1016/j.ijbiomac.2007.09.005

[16] J. C. Park, T. Ito, K. O. Kim, B. S. Kim, M. S. Khil and I. S. Kim, "Electrospun Poly(Vinyl Alcohol) Nanofibers:
Effects of Degree of Hydrolysis and Enhanced Water Stability," Polymer Journal, Vol. 42, No. 3, 2010, pp. 273-276. doi:10.1038/pj.2009.340

[17] O. Ohsawa, K. H. Lee, B. S. Kim, S. Lee and I. S. Kim, "Preparation and Characterization of Polyketone(PK) Fibrous Membrane via Electrospinning," Polymer, Vol. 51, No. 9, 2010, pp. 2007-2012. doi:10.1016/j.polymer.2010.02.045

[18] C. K. Kim, B. S. Kim, F. A. Sheikh, U. S. Lee, M. S. Khil and H. Y. Kim, "Amphiphilic Poly(Vinyl Alcohol) Hybrids and Electrospun Nanofibers Incorporating Polyhedral Oligosilsesquioxane," Macromolecules, Vol. 40, No. 14, 2007, pp. 4823-4828. doi:10.1021/ma070056e

[19] H. R. Kim, T. Ito, B. S. Kim, Y. Watanabe and I. S. Kim, "Mechanical Properties, Morphologies, and Microstructures of Novel Electrospun Metallized Nanofibers," $A d$ vanced Engineering Materials, Vol. 13, No. 5, 2011, pp. 376- 382. doi:10.1002/adem.201000320

[20] K. O. Kim, Y. A Seo, B. S. Kim, K. J. Yoon, M. S. Khil, H. Y. Kim and I. S. Kim, "Transition Behaviors and Hybrid Nanofibers of Ppoly(Vinyl Alcohol) and Polyethylene Glycol-POSS Telechelic Blends," Colloid and Polymer Science, Vol. 289, No. 8, 2011, pp. 863-870. doi:10.1007/s00396-011-2407-y

[21] K. O. Kim, B. S. Kim and I. S. Kim, "Self-Assembled Core-Shell Poly(Ethylene Glycol)-POSS Nanocarriers for Drug Delivery," Journal of Biomaterials Nanobiotechnology, Vol. 2, No. 3, 2011, pp. 201-206 doi:10.4236/jbnb.2011.23025

[22] Y. Osada and J. P. Gong, "Stimuli-Responsive Polymer gels and Their Application to Chemomechanical Systems," Progress in Polymer Science, Vol. 18, No. 2, 1993, pp. 187-226. doi:10.1016/0079-6700(93)90025-8

[23] B. S. Kim, J. P. Gong and Y. Osada, "Surfactant Binding by Polyelectrolyte Gels and Its Application to Electrodriven Chemomechanics," Polymer International, Vol. 48, 1999, pp. 619-698. doi:10.1002/(SICI)1097-0126(199908)48:8<691::AID-PI 203>3.0.CO;2-9

[24] X. Huang and C. S. brazel, "Analysis of Burst Release of Proxyphylline from Poly(vinyl alcohol) Hydrogels," Chemical Engineering Communications, Vol. 190, No. 4, 2003, pp. 519-532.

[25] P. J. Flory and J. J. Rehner, "Statistical Mechanics of Cross-Linked Polymer Networks," Journal of Chemical Physics, Vol. 11, No. 11, 1943, pp. 512-520. doi:10.1063/1.1723791

[26] P. Martens, J. Blundo, A. Nilasaroya, R. A. Odell, J. C. White and L. A. P. Warren, "Effect of Poly(vinyl alcohol) Macromer Chemistry and Chain Interactions on Hydrogel Mechanical Properties", Chemical Materials, Vol. 19, No. 10, 2007, pp. 2641-2648. doi:10.1021/cm0626381

[27] J. F. Kennedy, G. O. Phillips, P. A. Williams and V. Hascall, In: Hyaluronan, Ed., "Functions of Hyaluronan in Wound Repair," Woodhead Publishing Ltd, Cambridge, , 2002, pp. 341-348.

[28] J. Lesley, N. M. English, I. Gal, K. Mikecz, A. J. Day and 
R. Hyman, "Hyaluronan Binding Properties of a CD44 Chimera Containing the Link Module of TSG-6," Journal of Biological Chemistry, Vol. 277, No. 29, 2002, pp. 26600-26608. doi:10.1074/jbc.M201068200

[29] J. Lesley, I. Gal, D. J. Mahoney, M. R. Cordell, M. S. Rugg, R. Hyman, A. J. Day and K. Mikecz, "TSG-6 Modulates the Interaction Between Hyaluronan and Cell Surface CD44," Journal of Biological Chemistry, Vol.
279 , No. 24,2004 , pp. $25745-25754$. doi:10.1074/jbc.M313319200

[30] K. Wei, Y. Li, K. O. Kim, B. S. Kim, K. Abe, G. Q. Chen and I. S. Kim, "Fabrication of Nano-Hydroxyapatite on Electrospun Silk Fibroin Nanofiber and Their Effect in Osteoblastic Behavior," Journal of Biomedical Materials Research: Part A, Vol. 97A, No. 3, 2011, pp. 272-280. doi:10.1002/jbm.a.33054 